\title{
Réponses adaptatives des anguilles des régions tempérées à l'hétérogénéité environnementale et effets des pressions anthropiques
}

\section{Adaptive responses of eels of temperate regions to environmental heterogeneity and effects of anthropogenic pressures}

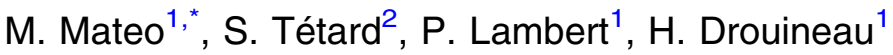 \\ 1 Irstea, Unité EABX-Écosystèmes Aquatiques et Changements Globaux, HYNES (Irstea-EDF R\&D), \\ 50 avenue de Verdun, 33612 Cestas Cedex, France mariamateosantos@gmail.com \\ 2 EDF Recherche et Développement, Laboratoire National d'Hydraulique et Environnement, HYNES \\ (Irstea-EDF R\&D), 6 quai Watier, 78401 Chatou Cedex, France
}

\begin{abstract}
Résumé - Le déclin mondial des anguilles tempérées est lié aux effets combinés de multiples pressions anthropiques. Cependant, la complexité du cycle de vie des anguilles et leurs capacités d'adaptation font qu'il est très difficile de connaître le poids relatif de chacune des pressions. Leurs panmixies et les longues dérives larvaires sont des freins aux adaptations locales, cependant on observe des patrons spatiaux de traits d'histoire de vie corrélés aux gradients environnementaux, à l'échelle du bassin versant et de son aire de répartition. Cette étude vise à (i) démontrer si ces patrons spatiaux d'histoire de vie sont le résultat de deux réponses adaptatives: le polymorphisme génétique et la plasticité phénotypique adaptative, et (ii) à réévaluer l'effet des différentes composantes du changement global en prenant en compte ces réponses adaptatives. Dans ce cadre, GenEveel, un modèle d'optimisation individu-centré a été développé et a permis de reproduire les patrons spatiaux observés concernant la longueur à l'argenture, le sex-ratio et la distribution des écotypes. Par la suite, différents types de pressions anthropiques (les pêcheries de civelles et d'anguilles argentées, les obstacles à la migration de montaison et les mortalités dues aux turbines hydroélectriques) ont été intégrés dans le modèle. L'objectif a été d'évaluer leurs impacts sur l'échappement (individus argentés sortant des eaux continentales), à la fois en nombre, mais aussi sur différents attributs comme le sex-ratio, la répartition entre génotypes, la longueur à l'argenture moyenne, et la production globale d'œufs. Les résultats montrent que la pression qui induit la plus forte mortalité directe sur les individus n'a pas forcément la plus forte influence sur la biomasse féconde et n'exerce pas nécessairement la pression sélective la plus forte sur les écotypes.
\end{abstract}

Mots-clés - Anguilla spp., mécanismes adaptatifs, changement global, pressions anthropiques, histoire de vie, modélisation 


\begin{abstract}
The worldwide decline of eels of temperate regions is due to a combination of several anthropogenic pressures. However, eels display very specific life-cycles and amazing adaptation capacities that impair our ability to assess the relative effects of each pressure. Their panmixia and the passive larval drifts also impair the possibility of local adaptation; however life history spatial patterns are correlated with environmental gradients at both river catchment and distribution area scales. This work aims (i) to explore whether these life history spatial patterns may result from two adaptive responses: genetic polymorphism and adaptive phenotypic plasticity, and (ii) to revisit the effect of different components of global change in consideration to these adaptive responses. In this context, GenEveel, an individual-based optimization model was developed. The model was able to mimic observed spatial patterns in length-at-silvering, sex ratio and distribution of ecotypes. Then, different types of anthropogenic pressures (glass eel fishery, silver eel fishery, obstacles to upstream migration, and mortality due to hydropower facilities) were integrated and the model was used to assess their impacts on the number of escapees and their attributes: sex ratio, repartition between genotypes, mean length at silvering, and overall egg production. The results showed that the pressure that induces the highest direct mortality has not necessarily the greatest influence on the spawning biomass and does not necessarily exert the strongest selective pressure on the ecotypes.
\end{abstract}

Keywords - Anguilla spp., adaptive mechanisms, global change, anthropogenic pressures, life history, modelling

\section{Introduction}

Différents mécanismes peuvent émerger comme réponse adaptative à l'hétérogénéité environnementale (Levins, 1963; Ernande \& Dieckmann, 2004). Cette question de l'adaptation à l'hétérogénéité de l'environnement est particulièrement intéressante pour les anguilles des régions tempérées. Ces trois espèces thalassotoques et panmictiques se reproduisent en mer - mer des Sargasses pour l'anguille européenne et l'anguille américaine (Schmidt, 1923; McCleave, 1993), à l'ouest des îles Mariannes pour l'anguille japonaise (Tsukamoto, 1992)mais passent leur phase de croissance dans des milieux extrêmement contrastés. En effet, après la reproduction, les larves sont transportées par les courants océaniques jusqu'aux eaux conti- nentales du Maroc à la Norvège pour l'anguille européenne (Tesch, 2003), du Venezuela au Groënland pour l'anguille américaine (Helfman et al., 1987) et du nord des Philippines à la Corée pour l'anguille japonaise (Tsukamoto, 1992). Ces particularités, panmixie et longue dérive larvaire, sont des freins à l'adaptation locale. Pour autant, des patrons d'histoire de vies corrélés aux gradients environnementaux sont observés à l'échelle de l'aire de répartition et à l'échelle des bassins versants (Tab. I) (Vladykov, 1966; Helfman et al., 1987; Vøllestad, 1992; Oliveira et al., 2001; Davey \& Jellyman, 2005; Kettle et al., 2011; Koops et al., 2014). Une hypothèse est que, malgré l'impossibilité d'adaptation locale, ces patrons soient liés pour partie à de la plasticité phénotypique qui pourrait être une réponse 
Tableau I. Principaux patrons de traits d'histoire de vie et d'attributs démographiques observées à l'échelle des bassins versants. Une flèche vers le haut indique une augmentation de l'aval vers l'amont et inversement pour une flèche vers le bas.

Table 1. Main patterns of life history traits and demographical attributes at the river catchment scale. An upward arrow indicates an increase from downstream to upstream and conversely for a downward arrow.

\begin{tabular}{|c|c|c|}
\hline $\begin{array}{l}\text { Trait/caractères } \\
\text { concerné }\end{array}$ & $\begin{array}{l}\text { Gradient } \\
\text { aval - amont }\end{array}$ & Référence \\
\hline Densité d'individus & & (Smogor et al., 1995; Ibbotson et al., 2002) \\
\hline Longueur à argenture & $\nearrow$ & (Helfman et al., 1984) \\
\hline $\begin{array}{l}\text { Taux de croissance } \\
\text { observés }\end{array}$ & $\searrow$ & $\begin{array}{l}\text { (Helfman et al., 1984; Melià et al., 2006a; } \\
\text { Daverat et al., 2012) }\end{array}$ \\
\hline Proportion de femelles & $\nearrow$ & (Oliveira \& McCleave, 2000 ; Tesch, 2003) \\
\hline $\begin{array}{l}\text { Proportion d'individus à } \\
\text { croissance lente }\end{array}$ & $\nearrow$ & (Boivin et al., 2015; Côté et al., 2015) \\
\hline
\end{tabular}

adaptative à l'hétérogénéité environnementale (Vøllestad, 1992; Daverat et al., 2006; Edeline, 2007; Geffroy \& Bardonnet, 2012; Drouineau et al., 2014), et à des polymorphismes génétiques soumis à de la sélection spatialement variable et à de la sélection d'habitat sous influence génétique (Côté et al., 2009, 2014, 2015; Pujolar et al., 2011; Gagnaire et al., 2012; Boivin et al., 2015; Pavey et al., 2015) (Fig. 1).

Comprendre les mécanismes adaptatifs qui expliquent la diversité phénotypique est essentiel pour la conservation et gestion des espèces (Brodersen \& Seehausen, 2014). Les populations d'anguilles sont en déclin depuis les années 1980-1990 (Dekker et al., 2003; Dekker \& Casselman, 2014), à tel point que l'anguille européenne est classée aujourd'hui en danger critique d'extinction dans la liste rouge de I'UICN (Jacoby \& Gollock, 2014a) et les anguilles américaine et japonaise sont classées comme en danger (Jacoby et al., 2014 ; Jacoby \& Gollock, 2014b). Plusieurs facteurs ont été proposés pour expliquer ce déclin (Jacoby et al., 2015), notamment des changements océaniques (Castonguay et al., 1994b), la contamination et la dégradation des habitats (Byer et al., 2015; Belpaire et al., 2016), le parasitisme (Feunteun, 2002), les pêcheries (Dekker, 2003b), la fragmentation et la perte massive de l'habitat (Kettle et al., 2011), et la mortalité induite par les turbines des centrales hydroélectriques (Castonguay et al., 1994a). Compte tenu de cette situation, les mesures de conservation se sont multipliées pour les trois espèces. Cependant, ces mesures de conservation consistent souvent à fixer une cible d'échappement pour les individus reproducteurs (en biomasse ou en proportion par rapport à une biomasse pristine) et à améliorer la colonisation et la production sur les bassins versants, comme c'est le cas pour le règlement européen anguille mis en place en 2007 pour reconstituer la population d'anguilles européennes (Règlement CE (CE) $n^{\circ} 1100 / 2007$ du Conseil). Ces mesures considèrent que tous les individus sont équivalents et ne prennent donc pas en compte le sexe, leur taille ou encore leur «qualité » (c'est-à-dire leur capacité à 


\section{A - adaptation locale, pas de panmixie}

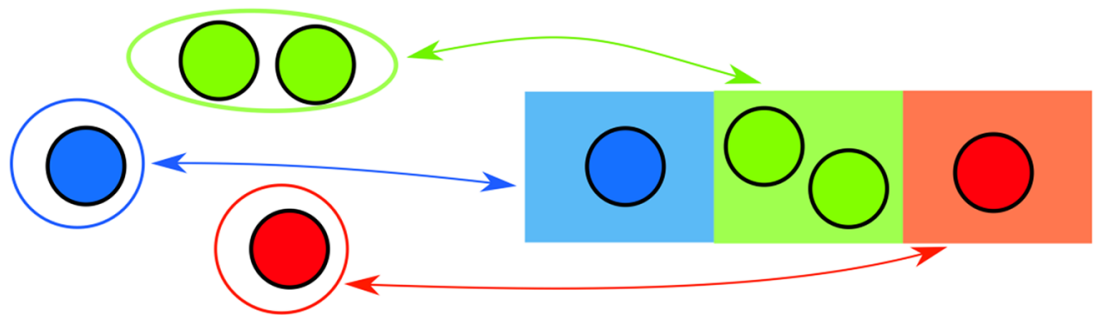

B - polymorphisme génétique et sélection spatialement variable avec panmixie

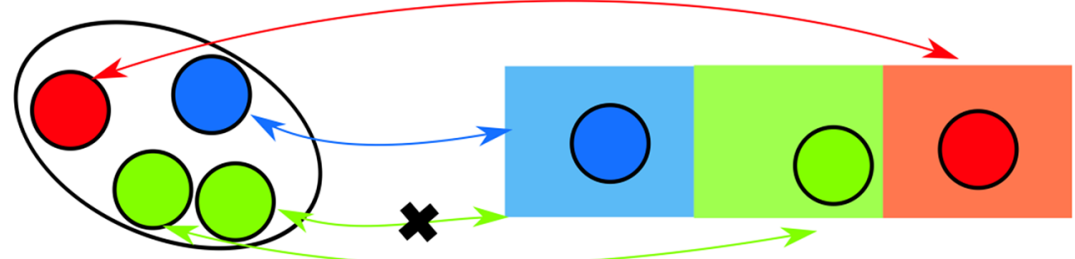

C - plasticité phénotypique adaptative avec panmixie

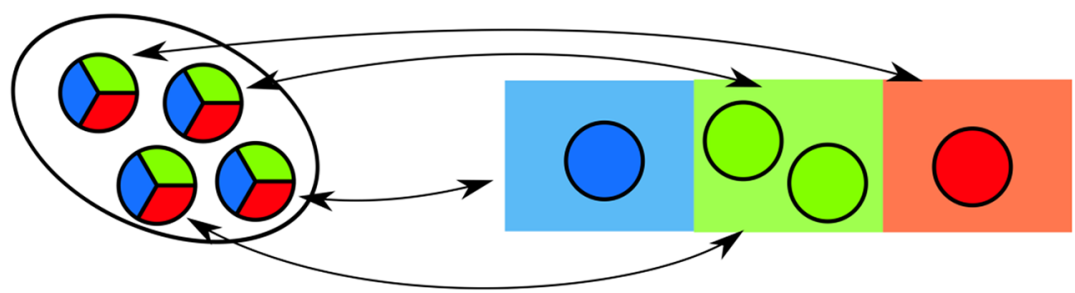

HABITAT(S) DE

HABITATS DE REPRODUCTION

CROISSANCE

Fig. 1. Représentations schématiques de trois mécanismes permettant de s'adapter à des environnements hétérogènes. Les cercles représentent des individus avec des génotypes particuliers (couleurs) dans des environnements spécifiques (rectangles). Par exemple, un individu jaune peut seulement survivre dans un habitat jaune, les phénotypes sont ainsi génétiquement déterminés et la sélection locale agit sur eux (A et B), ou bien un individu peut potentiellement exprimer différents phénotypes, et exprimera le phénotype correspondant à l'habitat en question, les phénotypes sont donc induits par la plasticité phénotypique sous pression de l'environnement (C). En cas de panmixie (B et $C)$, tous les individus se reproduisent ensemble, alors qu'en absence de panmixie, certains individus se reproduisent préférentiellement entre eux, pouvant permettre une adaptation locale (A) qui peut à terme mener à de la spéciation.

Fig. 1. Schematic representations of three adaptations that deal with heterogeneous environments. The circles represent individuals with particular phenotypes (colours) in specific environments (rectangles). For example, a yellow individual can only survive in a yellow habitat, and therefore these phenotypes are determined genetically and local selection acts on them (A and B), or an individual can potentially express the phenotypes, and will express the phenotype corresponding to its habitat, so the phenotypes are induced environmentally by phenotypic plasticity $(C)$. In case of panmixia (B and $C$ ), all individuals share a common reproduction whereas, in the absence of panmixia, some groups of individuals tend to mate preferentially within theirs groups, this last situation can lead to local adaptation $(A)$ and latter to speciation. 
contribuer à la génération suivante). Cependant, au regard des observations sur les variations des patrons d'histoire de vie corrélés aux gradients environnementaux (Tab. I), il apparaît important de s'interroger sur les attributs démographiques (sex-ratio, fécondité) des populations d'anguilles.

La forte hétérogénéité spatiale des pressions anthropiques sur les populations combinée à la variabilité phénotypique spatiale à l'échelle des bassins versants et de l'aire de répartition sont des défis majeurs pour la gestion de ces espèces. Dans le contexte du changement global et des déclins des trois espèces, l'objectif principal de ce travail était d'explorer l'écologie évolutive des anguilles tempérées pour comprendre les mécanismes impliqués dans leur capacité d'adaptation à la variabilité environnementale et la manière dont ces capacités interagissent avec les pressions anthropiques. Dans ce but, le fonctionnement des populations d'anguilles a été analysé afin (i) de mieux comprendre les mécanismes évolutifs impliqués dans l'adaptation à l'hétérogénéité environnementale et de (ii) quantifier les effets de pressions anthropiques en prenant en compte ces mécanismes pour améliorer la conservation des anguilles tempérées.

\section{Matériel et méthodes}

\subsection{GenEveel}

Une première approche de modélisation visant à comprendre les mécanismes évolutifs impliqués dans l'adaptation à l'hétérogénéité environnementale a été mise en œuvre.
GenEveel (Genetics \& Evolutionary Ecology-based model for Eel), la nouvelle version d'EvEel (Drouineau et al., 2014), est un modèle de type individucentré. Le modèle repose sur un postulat d'optimisation de la fitness - la fitness étant définie en écologie évolutive comme la capacité d'un individu à transmettre ses gènes aux générations suivantes - et vise à explorer le rôle des mécanismes adaptatifs dans l'émergence des patrons spatiaux d'histoire de vie chez l'anguille (Tab. I). Comme dans EvEel, le modèle utilise la probabilité de survie jusqu'à une longueur à argenture prédéterminée comme un proxy de la fitness des mâles. En effet, les mâles sont supposés suivre une tactique de «minimisation du temps », c'est-à-dire de partir vers la zone de reproduction dès qu'ils ont atteint la taille minimale garantissant le succès de la migration (Vøllestad, 1992). Au contraire, les femelles sont supposées suivre une stratégie de maximisation de la taille, c'est-à-dire de recherche d'un compromis entre une plus grande taille qui permet d'augmenter la fécondité et une probabilité de survie qui diminue pour atteindre ces grandes tailles (Helfman et al., 1987). Dans ce cadre, la fitness des femelles est calculée dans le modèle comme le produit de la fécondité à la longueur à argenture multipliée par la probabilité de survivre jusqu'à cette taille.

Suite aux observations de Côté et al. (2015) de patterns génétiques des taux de croissance selon l'origine des anguilles, le modèle ajoute à EvEel, qui intégrait déjà la plasticité phénotypique, un polymorphisme génétique sur la croissance. Cela se fait via la 


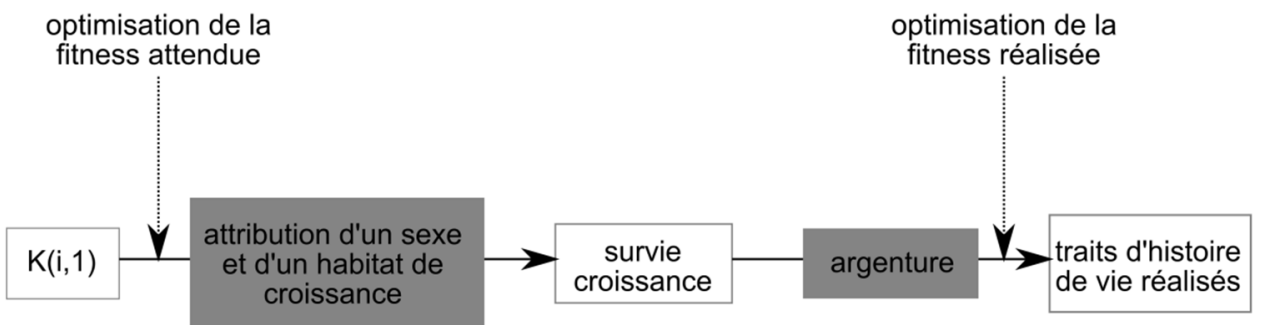

Fig. 2. Séquence du modèle GenEveel représentant les étapes biologiques successives.

Fig. 2. Flow chart of GenEveel representing the fish biological pathway.

distinction entre des individus à croissance lente et des individus à croissance rapide. Ainsi, chaque individu est caractérisé par un taux de croissance intrinsèque $\left(K_{i}\right)$; en cherchant à maximiser la fitness espérée, il détermine son sexe, sélectionne son habitat de croissance dans un bassin versant, puis mature à une longueur optimale. Le bassin versant a été schématiquement représenté par une séquence de cellules de même taille, la première cellule représentant l'estuaire, la n-ième représentant la source de la rivière. Compte-tenu du fait qu'il est observé un taux de croissance plus élevé dans les zones salées à l'aval, que dans les zones d'eau douce plus en amont (Acou et al., 2003; Melià et al., 2006b; Edeline, 2007; Cairns et al., 2009; Côté et al., 2009; Daverat et al., 2012), le taux de croissance réalisé dans une cellule dépend à la fois du taux de croissance intrinsèque des individus et de la position dans le bassin versant (c'està-dire la cellule). La mortalité naturelle réalisée dépend à la fois des taux de mortalité naturelle intrinsèques, de la position dans le bassin versant, et du nombre d'individus dans le bassin versant (de façon à intégrer une mortalité naturelle densité-dépendante [Vøllestad \& Jonsson, 1988; De Leo \& Gatto, 1995 ; Lobón-Cerviá \& Iglesias, 2008; Bevacqua et al., 2011b]).

Le modèle présente deux étapes principales (Fig. 2). Dans une première étape, les individus sélectionnent leur habitat de croissance (cellule) et déterminent leur sexe (mâle ou femelle), individu après individu (ordre aléatoire). Pour ce faire, ils "sélectionnent» la combinaison habitat-sexe qui leur garantirait la fitness maximum étant donné les choix déjà réalisés par les individus précédents. Une fois cette étape réalisée pour tous les individus, la longueur à l'argenture optimale est estimée pour toutes les femelles (les mâles ayant une longueur à l'argenture constante) étant données les positions des poissons après l'étape 1 , et le modèle calcule ensuite les taux de survie jusqu'à l'argenture, qui sont multipliés par la fécondité pour les femelles (fonction de la longueur à argenture), et par un paramètre dit de fertilité pour les mâles qui permet de donner des grandeurs comparables aux fitness mâles et femelles. Les étapes et sous étapes du modèle et les mises en équation sont précisément décrites dans Mateo et al. (2017a). 
Dans un premier temps, on a vérifié que les patrons spatiaux (Tab. I) sont bien reproduits quand on utilise le meilleur jeu de paramètres donné par la littérature (Tab. II). Des tests de tendance de Mann-Kendall ont été réalisés sur chacun des patrons pour détecter une tendance monotone croissante ou décroissante sur la variable d'intérêt et ainsi confirmer les patrons spatiaux observés. Le signe du coefficient de corrélation calculé donne l'évolution de la tendance sur le bassin versant (une valeur positive indique une tendance à la hausse vers l'amont et inversement pour une valeur négative) et sa valeur indique l'intensité de la corrélation.

Une exploration numérique a été ensuite réalisée pour déterminer dans quelles combinaisons de paramètres tous les patrons spatiaux documentés sont reproduits à l'échelle des bassinsversants (abondance plus forte à l'aval, longueur à l'argenture plus élevée en amont, proportion de femelles plus forte en amont, proportion d'individus à croissance rapide plus forte à l'aval et taux de croissance réalisé plus rapide à l'aval-Tab. I). Pour cela, un plan de simulation factoriel fractionnaire a été mis en œuvre (i) pour apprécier la sensibilité des patrons simulés aux valeurs prises par les différents paramètres et (ii) pour mieux comprendre dans quels types de conditions les patrons étaient correctement reproduits. Pour chacun des paramètres, une valeur haute et une valeur basse avec $20 \%$ de variation autour de la valeur de référence ont été fixées pour effectuer les simulations. De nouveau, des tests de tendance de Mann-Kendall ont été réalisés pour chacune des variables d'intérêt sur chacune des simulations.

\subsection{Impact des pressions anthropiques}

Par la suite, GenEveel a été utilisé pour comprendre la répercussion des pressions anthropiques sur la population. En effet, les pressions anthropiques, si elles peuvent être une source de mortalité directe, peuvent également affecter les traits d'histoire de vie (taille à maturité) et les attributs démographiques (sex-ratio) en jouant sur les effectifs et donc les mécanismes densité-dépendants ou sur les habitats accessibles, voir en affectant plus certains écotypes que d'autres. Les conséquences de ces pressions seront différentes selon la nature des mécanismes adaptatifs qui modifient les caractères des individus : en favorisant des individus à croissance rapide (ou lente), en modifiant le sex-ratio, la longueur à l'argenture ou la distribution spatiale.

Ainsi, GenEveel a été adapté pour intégrer l'effet des pressions anthropiques au niveau des processus de colonisation et de dévalaison afin d'en quantifier les conséquences sur l'échappement des anguilles argentées (individus argentés sortant des eaux continentales), non seulement en termes de nombre d'anguilles, mais aussi l'effet sur le sex-ratio, les proportions des individus à croissance lente, la longueur à l'argenture et la production d'œufs, après une génération. Le modèle a été structuré en représentant les événements et les processus du cycle de vie de l'anguille en milieux continentaux (en utilisant les paramètres de référence de la littérature) et en intégrant 


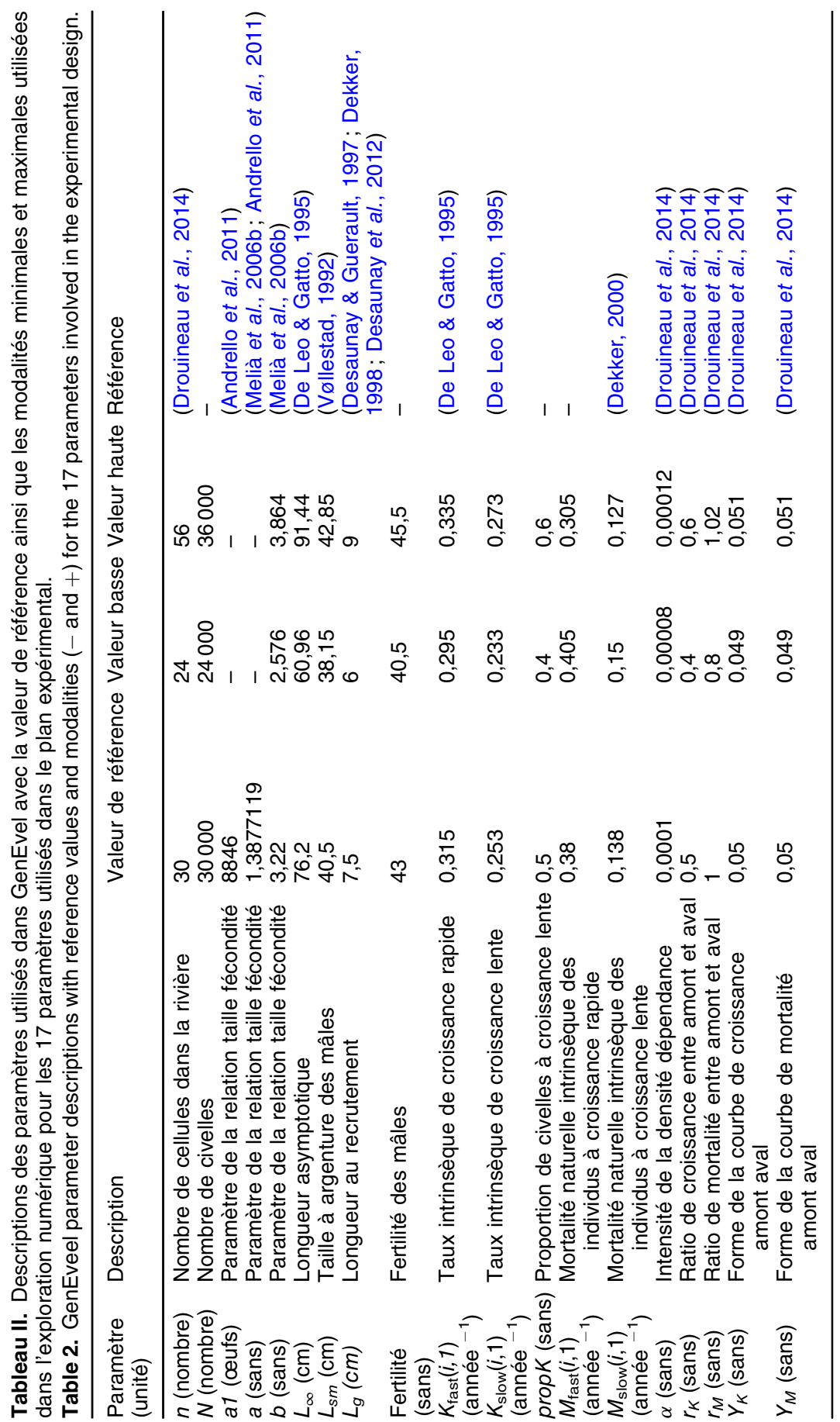




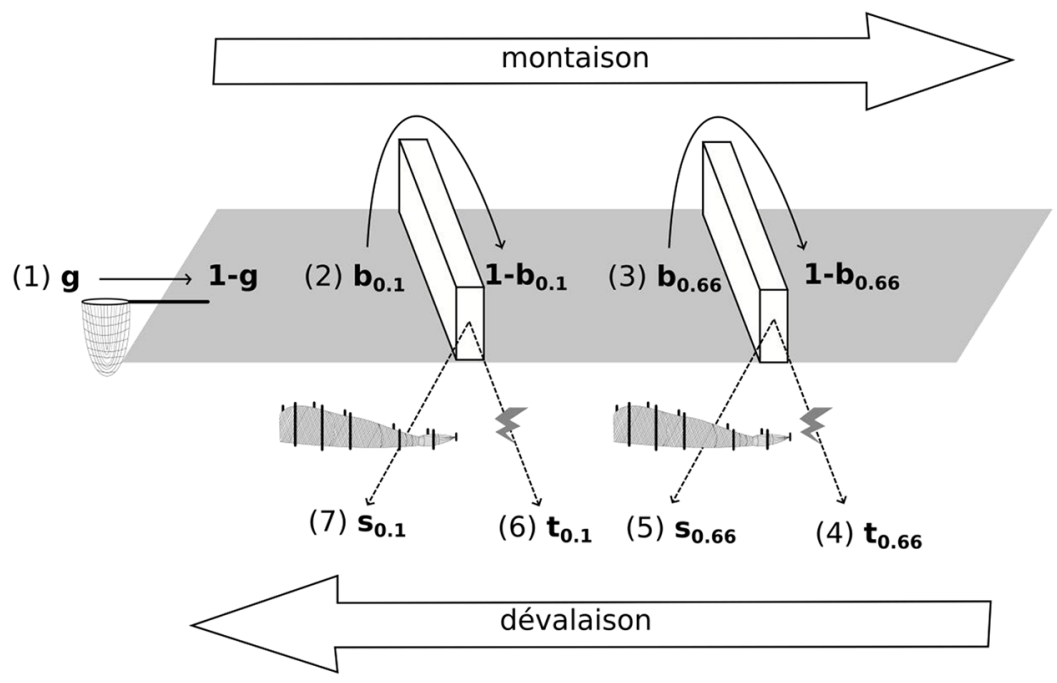

Fig. 3. Représentation conceptuelle du bassin versant, indiquant les sept pressions anthropiques considérées dans l'étude: (1) $g$ : taux de capture de la pêcherie civelière, (2) $b_{0,66}$ et (3) $b_{0,1}$ : taux de blocage des obstacles (localisés près de la source et près de l'estuaire, arbitrairement fixés au premier et au deuxième tiers du bassin versant, respectivement), (4) $t_{0,66}$ et $(6) t_{0,1}$ : taux de mortalité par les turbines (localisées près de la source et près de l'estuaire, arbitrairement fixés au premier et au deuxième tiers du bassin versant, respectivement), (5) $s_{0,66}$ et (7) $s_{0,1}$ : taux de capture par les pêcheries d'anguilles argentées (localisées près de la source et près de l'estuaire, arbitrairement fixés au premier et au deuxième tiers du bassin versant, respectivement).

Fig. 3. Conceptual representation of the catchment, indicating the seven anthropogenic pressures considered in the study: (1) $g$ : catch rate by the glass eel fishery, (2) $b_{0,66}$ and (3) $b_{0,1}$ : blockage rates (located near the river source and the river mouth, fixed at one third and two thirds of the catchment, respectively), (4) $t_{0,66}$ and (6) $t_{0,1}$ : turbine mortality rates (located near the river source and the river mouth, fixed at one third and two thirds of the catchment, respectively), (5) $s_{0,66}$ and (7) $s_{0,1}$ : catch rates by the silver eel fisheries (located near the river source and the river mouth, fixed at one third and two thirds of the catchment, respectively).

les effets des pressions anthropiques dans les simulations (Fig. 3). Tel qu'implémenté dans le modèle (Figs. 2 et 3 ), l'impact des barrages et les pêcheries arrivent très tôt dans le cycle de vie et peuvent donc impacter le choix de l'habitat de croissance et la détermination du sexe, ainsi que la croissance et la survie durant la phase anguille jaune. Les pêcheries argentées et turbines n'impactent elles que les anguilles ayant survécu jusqu'à la maturation. Cinq indicateurs ont été calculés pour toutes les simulations à partir des anguilles ayant survécu au processus :
- l'échappement $(N s)$;

- la longueur moyenne à l'argenture $(L s)$;

- la proportion de femelles (sex-ratio) $(\mathrm{Sr})$;

- la production d'œufs (somme de la fécondité des femelles parmi les survivants) $(E)$;

- la proportion d'individus à croissance lente $(S I)$.

II est à noter que nous n'avons pas considéré l'effet de toutes les pressions anthropiques impactant l'anguille dans cette étude. Si la destruction d'habitat 
peut être «représentée » par la situation d'un barrage infranchissable, la pêcherie d'anguilles jaunes, la pollution ou l'effet d'Anguillicola crassus n'ont pas été étudiés pour différentes raisons. Premièrement, car GenEveel ne décrit pas la phase de croissance à proprement parler et donc il est difficile de prendre en compte les mortalités ciblant ce stade. Deuxièmement, car si pêche argentée, civelière ou barrage ont lieu en des points précis d'un bassin versant et à un moment spécifique du cycle de vie, pollution, pêcherie jaune et parasitisme sont plus diffus dans l'espace et dans le temps et donc difficiles à représenter. Enfin, concernant parasitisme et pollution, il existe assez peu d'information quantitative sur leurs effets sur la dynamique des populations.

Une exploration numérique de l'espace des paramètres a ensuite été réalisée. Pour cela, un plan de simulations de type hypercube latin (Iman \& Conover, 1980 ; McKay, 1988; McKay et al., 2000) a été mis en œuvre. Pour chacune des 10000 simulations, les cinq indicateurs présentés précédemment ont été calculés. À l'issue de ces simulations, et ce pour chacun des cinq indicateurs considérés, un modèle additif généralisé a été ajusté en utilisant l'indicateur comme variable à expliquer et les pressions anthropiques $\left(g, b_{0,66}, b_{0,1}, s_{0,66}, s_{0,1}, t_{0,66}, t_{0,1}\right)$ comme variables explicatives (Fig. 3 ). Des graphiques représentant les effets marginaux ont été utilisés pour explorer les relations entre les pressions et les variables de sortie. Les signes des coefficients de régression ont permis d'indiquer si la pression avait un impact positif ou négatif sur l'indicateur considéré. Enfin, les coefficients de corrélation de rang partiels (PRCCPartial Rank Correlation Coefficient [Saltelli et al., 2000]) ont été calculés pour évaluer les effets globaux des pressions anthropiques sur chacune des variables de sortie. Ils permettent de déterminer le degré d'association entre un prédicteur et une variable après avoir omis l'effet des autres prédicteurs.

\section{Résultats}

\subsection{GenEveel}

Les résultats détaillés ont été présentés par Mateo et al. (2017a). En utilisant les paramètres de la littérature, le modèle est capable de reproduire les patrons spatiaux observés dans les bassins versants : à la fois ceux sur les traits de vie, mais aussi ceux sur la distribution des génotypes (Fig. 4). Les mâles sont concentrés dans la partie aval de la rivière où la densité d'individus est supérieure (Helfman et al., 1987 ; Tesch, 2003 ; Davey \& Jellyman, 2005). Les individus à croissance rapide s'installent préférentiellement dans les habitats aval, alors que les individus à croissance plus lente ont tendance à s'installer à l'amont pour éviter la compétition (De Leo \& Gatto, 1995; Daverat et al., 2006, 2012; Edeline, 2007; Geffroy \& Bardonnet, 2012). Concernant la longueur à l'argenture (des mâles et des femelles), une plus petite taille à maturité est simulée dans la partie aval de la rivière, alors que des longueurs plus importantes sont retrouvées au fur et à mesure de la progression vers l'amont (Vøllestad, 1992; Oliveira, 1999). 

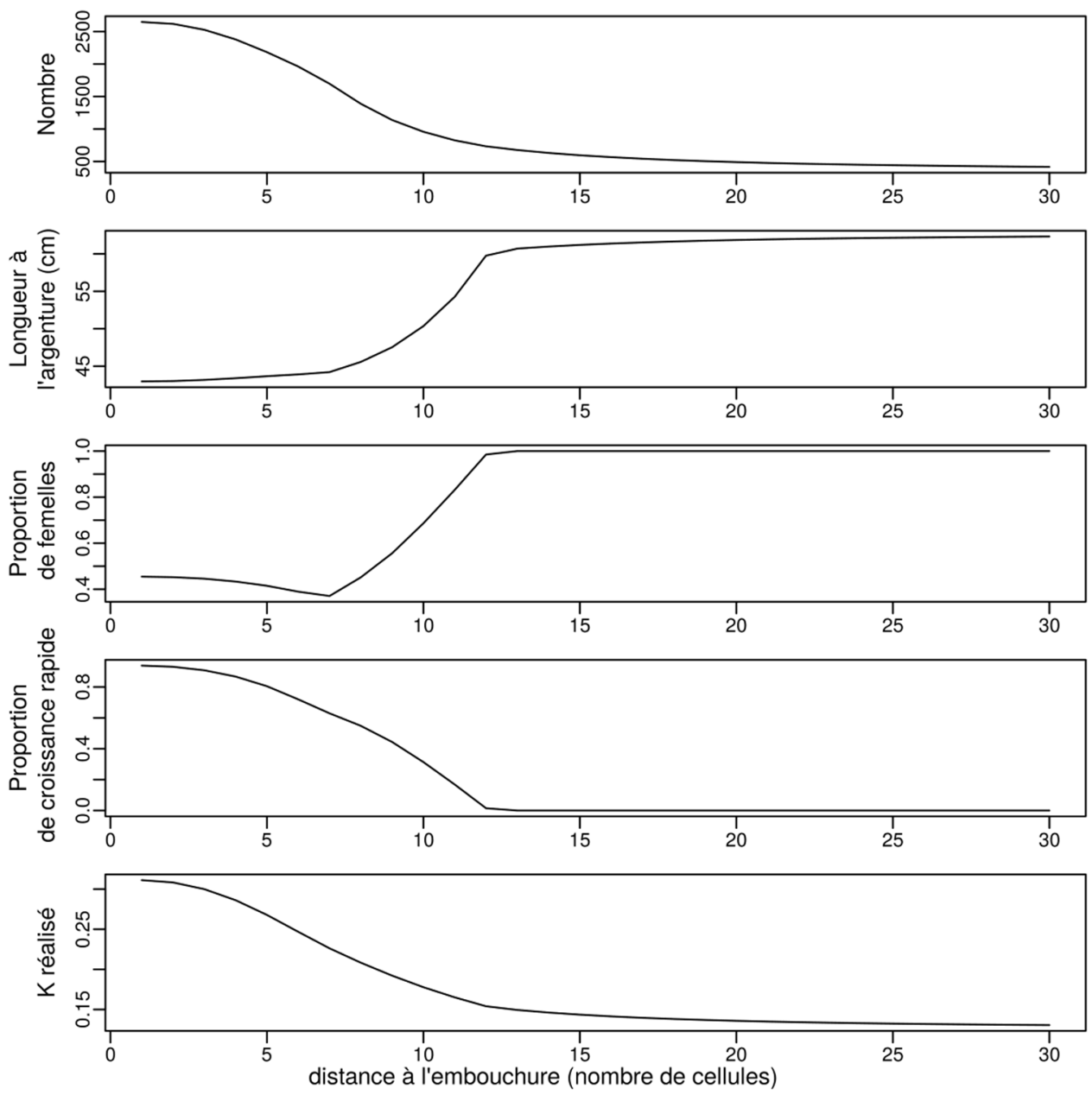

Fig. 4. Variables de sortie représentant les cinq patrons spatiaux résultant de la simulation de GenEveel en utilisant les paramètres de référence donnés par la littérature.

Fig. 4. Outputs values for the five spatial patterns resulting from the reference simulation.

À l'issue des simulations faisant varier la valeur des paramètres, 130 simulations (13 combinaisons de paramètres) sur 2560 ont reproduisent les 5 patrons spatiaux (Tab. III). Dans les situations où la fitness des femelles était trop favorisée en raison d'une forte longueur asymptotique $L_{\infty}$ ou d'un fort para- mètre de fertilité, le modèle n'a produit que des femelles. À l'inverse, dans des situations où les femelles étaient trop pénalisées, le modèle n'a produit que des mâles. Un équilibre entre mâles et femelles est donc requis pour reproduire les patrons spatiaux. Les patrons concernant la longueur à l'argenture et le sex-ratio 
Tableau III. Paramétrisation des 13 combinaisons ayant correctement généré les 5 patrons spatiaux. Le signe $+/$ - correspond aux modalités prises par les groupes de paramètres (signe + , modalité $+20 \%$ de la valeur de référence; signe -, modalité $-20 \%$ de la valeur de référence). Chaque ligne correspond à une combinaison. Chaque variable est précisément définie dans le tableau II.

Table 3. Parametrization of the 13 combinations that generated five consistent patterns. The signs $+/-$ refer to the modalities of the parameters groups (sign + , modality $+20 \%$ of reference value; sign -, modality $-20 \%$ of reference value). Each row corresponds to a combination.

\begin{tabular}{|c|c|c|c|c|c|c|c|c|c|c|c|}
\hline$N$ & $\begin{array}{l}\text { Fertility } \\
\text { Lsm }\end{array}$ & $\begin{array}{l}\text { Kfast }(i, 1) \\
\text { Mfast }(i, 1)\end{array}$ & $\begin{array}{l}\text { Kslow }(i, 1) \\
\text { Mslow }(i, 1)\end{array}$ & propK & $\alpha$ & $n$ & $b$ & $L_{\infty}$ & $L_{g}$ & $\begin{array}{l}r_{K}, \\
r_{M}\end{array}$ & $\begin{array}{l}\gamma_{K} \\
\gamma_{M}\end{array}$ \\
\hline - & - & - & + & + & + & + & + & - & - & + & + \\
\hline+ & - & - & + & - & + & + & + & - & + & + & + \\
\hline+ & - & - & + & + & - & + & + & - & + & + & - \\
\hline- & - & - & + & - & - & + & + & - & - & + & - \\
\hline+ & - & + & + & + & - & - & + & - & - & + & - \\
\hline+ & - & - & + & - & - & - & + & - & + & - & - \\
\hline- & - & - & + & - & + & + & - & + & + & + & + \\
\hline- & - & + & + & - & - & - & + & - & + & + & - \\
\hline- & - & + & + & + & + & - & + & - & + & + & + \\
\hline- & - & + & + & - & + & + & + & - & + & - & + \\
\hline+ & - & + & + & - & + & - & + & - & - & + & + \\
\hline- & - & - & + & - & + & - & + & - & - & - & + \\
\hline+ & - & + & + & - & - & + & + & - & - & - & - \\
\hline
\end{tabular}

se sont avérés être les deux patrons les plus contraignants à reproduire et les plus sensibles à certains paramètres (longueur asymptotique, paramètre $b$ de la relation taillefécondité, fertilité des mâles, Iongueur à l'argenture des mâles). Ces paramètres positionnent l'équilibre entre la fitness des mâles et celle des femelles (fertilité des mâles et longueur à l'argenture, $b$ et $L_{\infty}$ ) et les avantages relatifs entre individus à croissance lente et rapide. L'exploration numérique montre que ces patrons sont respectés à condition que les individus à croissance lente et les femelles ne soient pas trop pénalisés par rapport aux individus à croissance rapide et aux mâles.

\subsection{Impact des pressions anthropiques}

Les résultats détaillés ont été présentés par Mateo et al. (2017b).
Les PRCC sont synthétisés dans le tableau IV et les GAMs sur la figure 5. La pêcherie civelière et l'obstacle à la montaison ont le plus d'influence sur l'échappement, la taille à argenture et la proportion de femelles. De façon intéressante, en capturant des individus, la pêcherie civelière diminue le nombre de survivants, mais la baisse de densité qui en découle fait qu'une plus forte proportion d'individus devient femelles et atteignent de plus grande longueur à argenture. Au contraire, l'obstacle contraint mais ne tue pas de civelles et les pousse à rester dans des milieux à croissance forte et à forte densité. Plus d'individus deviennent donc mâles et partent à de faibles longueurs à l'argenture.

Si les pêcheries d'anguilles argentées et les turbines hydroélectriques ont un effet plus faible (mais négatifs) sur ces trois sorties (échappement, taille à l'argenture et proportion de femelles), quand elles sont situées à 
Tableau IV. Coefficients de corrélation de rang partiels entre les variables de sortie (lignes) et les pressions (colonnes). Un coefficient positif indique une corrélation positive. Ns: échappement d'anguilles argentées (nombre), Ls: Iongueur moyenne à l'argenture, SR: proportion de femelles (sexratio), E : quantité d'œufs produite, SI : proportion d'individus à croissante lente, $g$ : pêcherie civelière, $s_{0,66}$ : pêcherie d'anguilles argentées située à l'amont, $s_{0,1}$ : pêcherie située à l'aval, $t_{0,66}$ : turbines situées à l'amont, $t_{0,1}$ : turbines situées à l'aval, $b_{0,66}$ and $b_{0,1}$ : obstacles à la montaison situés respectivement à l'amont et à l'aval.

Table 4. Partial rank correlation coefficients between output variables (rows) and pressures (columns). A positive coefficient indicates a positive correlation. Ns: number of escapees, Ls: mean length-atsilvering, SR: proportion of females (sex-ratio), E: egg production, Sl: proportion of slow growers, $g$ : glass eel fishery, $s_{0,66}$ : silver eel fishery located near the river source, $s_{0,1}$ : silver eel fishery located near the river mouth, $t_{0,66}$ : turbine located near the river source, $t_{0,1}$ : turbine located near the river mouth, $b_{0,66}$ and $b_{0,1}$ : obstacles to upstream migration located near the river source and mouth respectively.

\begin{tabular}{lrrrrrrr}
\hline & $\mathrm{g}$ & $\mathrm{b}_{0,1}$ & $\mathrm{~b}_{0,66}$ & $\mathrm{~s}_{0,1}$ & $\mathrm{~s}_{0,66}$ & $\mathrm{t}_{0,1}$ & $\mathrm{t}_{0,66}$ \\
\hline Ns & $-0,95$ & 0,77 & 0,02 & $-0,39$ & $-0,02$ & $-0,39$ & $-0,01$ \\
Ls & 0,94 & $-0,75$ & 0 & $-0,33$ & 0 & $-0,33$ & $-0,02$ \\
SR & 0,95 & $-0,57$ & 0,01 & $-0,14$ & 0,01 & $-0,12$ & $-0,01$ \\
E & $-0,49$ & $-0,01$ & 0,01 & $-0,42$ & $-0,01$ & $-0,4$ & $-0,01$ \\
SI & 0,22 & 0,88 & 0,02 & $-0,35$ & $-0,03$ & $-0,35$ & $-0,01$ \\
\hline
\end{tabular}

l'aval, elles ont en revanche un effet sur la production d'œufs presque aussi fort que la pêcherie civelière et plus fort que les obstacles à la montaison. Cela s'explique par des mécanismes de compensation: la perte d'échappement liée à la pêcherie civelière est compensée par une plus forte proportion de femelles qui sont de plus grandes tailles, ce qui permet d'atténuer la baisse de production d'œufs. Pour l'obstacle à la montaison, la hausse de l'échappement est compensée par une plus forte production de mâles et une taille à argenture plus faible. Au contraire, la pêcherie d'argentées et les turbines agissant après la détermination du sexe et le choix de l'habitat, il n'y a pas de mécanismes de compensation pour ces pressions. De plus, ils affectent les individus situés à l'amont, qui sont plus majoritairement des femelles de grande taille et donc importantes pour la production d'œufs.

Finalement, la pêcherie d'anguilles argentées et les turbines localisées à l'aval ont diminué la proportion d'indi- vidus à croissance lente parce que ces individus ont tendance à s'installer dans les habitats situés à l'amont et étaient en conséquence plus impactés que les individus à croissance rapide (Tab. IV - Fig. 5). D'autre part, l'obstacle situé à l'aval a entraîné l'augmentation de la proportion d'individus à croissance lente; ces individus étaient bloqués dans des habitats aval où la croissance était plus rapide, résultant en une meilleure survie jusqu'à l'argenture (comme observé pour l'échappement-Fig. 5). Cependant, les individus bloqués étaient «contraints " de rester dans les habitats aval, résultant ainsi en une diminution de leur fitness et une forte compétition avec les individus à croissance rapide. À ce titre, ces pressions anthropiques peuvent constituer des pressions de sélection favorisant les individus à croissance rapide au détriment des individus à croissance lente. De façon intéressante, la pêcherie civelière, qui exploite les individus à croissance lente et rapide de manière équivalente, a 

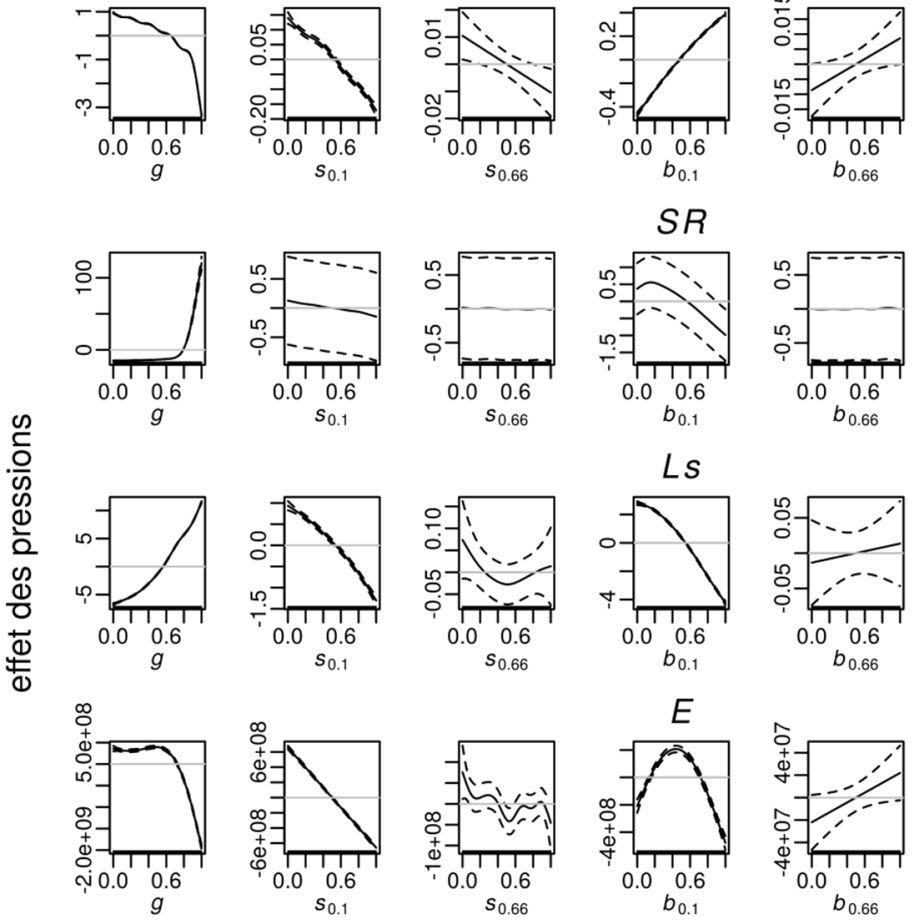

\section{SI}
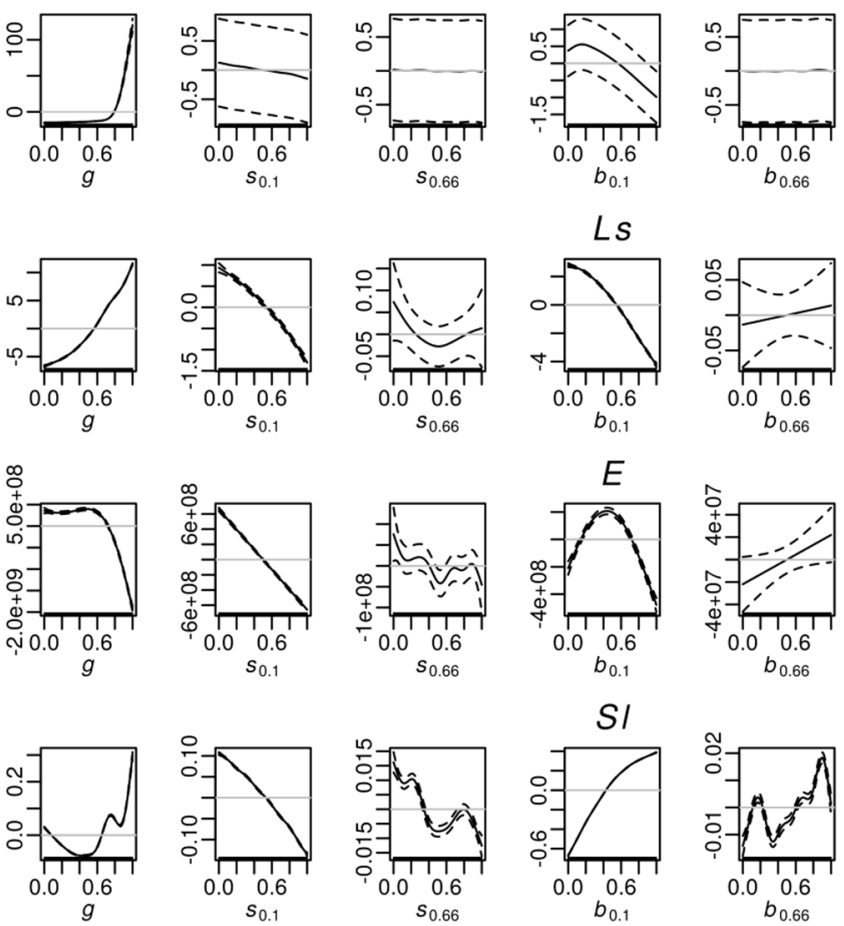
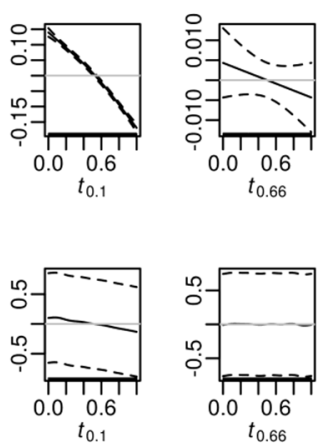

intensité des pressions
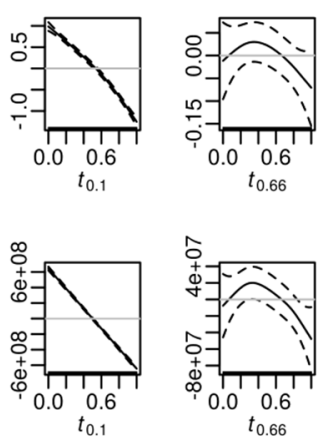

Fig. 5. Effets des pressions sur les cinq indicateurs estimés par les GAMs. Ns: échappement d'anguilles argentées (nombre), SR: proportion de femelles (sex-ratio), Ls: Iongueur moyenne à l'argenture, E: quantité d'œufs produite, SI: proportion d'individus à croissante lente, $g$ : pêcherie civelière, $s_{0,66}$ et $s_{0,1}$ : pêcheries d'anguilles argentées situées à l'amont et à l'aval, respectivement, $t_{0,66}$ et $t_{0,1}$ : turbines situées à l'amont et à l'aval, respectivement, $b_{0,66}$ et $b_{0,1}$ : obstacles à la montaison situés à l'amont et à l'aval, respectivement.

Fig. 5. Effects of the pressures on the five indicators estimated by the GAMs. Ns: number of escapees, SR: proportion of females (sex-ratio), Ls: mean length-at-silvering, E: egg production, SI: proportion of slow growers, $g$ : glass eel fishery, $s_{0,66}$ and $s_{0,1}$ : silver eel fisheries located near the river source and the river mouth, respectively, $t_{0,66}$ and $t_{0,1}$ : turbines located near the river source and the river mouth, respectively, $b_{0,66}$ and $b_{0,1}$ : obstacles located near the river source and the river mouth, respectively.

tendance à favoriser les individus à croissance rapide pour un faible taux d'exploitation et les individus à croissance lente pour un plus fort taux d'exploitation (à fort taux d'exploitation, la très faible mortalité dépendante ayant tendance à fortement favoriser les individus à croissance lente). 


\section{Discussion}

Malgré le grand intérêt pour les anguilles tempérées et la littérature scientifique sur des aspects particuliers et la dynamique de population de ces espèces (De Leo \& Gatto, 1995; Dekker, 2000; Davey \& Jellyman, 2005 ; Bevacqua et al., 2011b; Geffroy \& Bardonnet, 2016), la compréhension des mécanismes adaptatifs des anguilles face à l'hétérogénéité environnementale a connu des avancées très récentes (Daverat et al., 2006; Edeline, 2007; Gagnaire et al., 2012; Drouineau et al., 2014; Boivin et al., 2015; Pavey et al., 2015). Depuis longtemps, de nombreuses études ont montré des corrélations entre les traits d'histoire de vie et des gradients environnementaux (Vélez-Espino \& Koops, 2009), comme la longueur à l'argenture (Helfman et al., 1987; Jessop, 2010), le sex-ratio (Davey \& Jellyman, 2005; Kettle et al., 2011), et les effets de la densité-dépendance (Vøllestad \& Jonsson, 1988; Bevacqua et al., 2011a). Récemment, d'autres études expérimentales ont mis en évidence la base génétique de la variation des traits d'histoire de vie comme la croissance et des corrélations entre les conditions environnementales et l'expression génétique (Gagnaire et al., 2012; Pujolar et al., 2014; Boivin et al., 2015; Côté et al., 2015; Pavey et al., 2015). Cependant, peu d'études ont analysé toutes ces observations simultanément afin de trouver une signification adaptative commune.

Nos résultats suggèrent que la sélection de l'habitat dépendant du génotype (par le biais d'un polymor- phisme génétique) et la plasticité phénotypique peuvent être deux mécanismes adaptatifs complémentaires qui permettent à l'anguille de faire face à un environnement hétérogène. Le choix de l'habitat de croissance est le résultat du croisement entre les caractéristiques de l'environnement, le génotype de l'individu et la compétition intraspécifique. La plasticité phénotypique joue un rôle important dans la variabilité des traits d'histoire de vie et d'attributs démographiques induits par l'environnement. Nos résultats aident à la compréhension de différentes stratégies de vie selon le sexe. Ils suggèrent que les individus choisiront leur stratégie selon leur croissance, qui elle-même présente une base génétique. Les individus à croissance rapide ont une mortalité plus élevée et choisissent préférentiellement des habitats à forte densité, une stratégie du temps minimal est favorisée. Les individus à croissance lente ont une mortalité intrinsèque plus faible et choisissent des habitats à plus faibles densités ce qui favorise une stratégie de taille maximale.

L'effondrement des populations d'anguilles tempérées a entraîné la mise en place de mesures visant à diminuer toutes les sources de mortalité anthropiques. Au niveau européen, des plans de gestion à l'échelle des états membres ont été mis en place et se focalisent naturellement sur les taux de mortalité induits par les pressions et sur le taux d'échappement des anguilles. La question immédiate qui se pose est de comprendre la répercussion des pressions anthropiques sur la population, car si elles peuvent être une source 
de mortalité accrue, elles peuvent également affecter les traits d'histoire de vie (taille à maturité) et les attributs démographiques (sex-ratio) en jouant sur les effectifs et donc les mécanismes densité-dépendants ou sur les habitats accessibles, voir en affectant plus certains types d'individus que d'autres.

Nos résultats après intégration des pressions anthropiques dans GenEveel montrent que les impacts des dites pressions sont complexes et que ce n'est pas forcément la pression qui tue le plus grand nombre d'anguilles qui a le plus gros impact sur la biomasse féconde ou la production d'œufs. D'autre part, un résultat important de cette étude est que l'impact des pressions anthropiques dépend clairement de leur position dans les bassins versants. Bien que cela soit relativement évident, les conséquences à l'échelle de la population d'anguille le sont moins car les pressions anthropiques ne sont pas distribuées uniformément. Par exemple, en Europe, des centrales hydroélectriques peuvent être implantées à l'aval des bassins versants comme en Scandinavie par exemple, alors qu'elles sont davantage situées en amont en France. Les pêcheries d'anguilles argentées sont principalement localisées en Scandinavie et autour de la mer Méditerranée (Dekker, 2003a), alors que les pêcheries civelières sont plutôt présentes en France et en Espagne. Ainsi, puisque les patrons de traits d'histoire de vie et les différences de densité sont aussi observés à l'échelle de l'aire de répartition, il est très difficile de dresser un panorama global de l'effet de chaque pression anthropique à l'échelle de la population.
Nos résultats démontrent surtout que la plasticité permet de compenser les effets de certaines pressions (pêcherie civelière et obstacles à la montaison) et est source de résilience pour la population, alors que d'autres pressions ne bénéficient d'aucun effet de compensation (pêcherie argentée et turbines à la dévalaison). La plupart des pressions, sauf la pêcherie civelière, favorisent les individus à croissance rapide par rapport aux individus à croissance lente, démontrant que les pressions anthropiques peuvent être des pressions sélectives pour la population d'anguilles. Compte tenu du désavantage évolutif apparent des individus à croissance lente, on peut se demander comment ces individus ont pu être conservés par la sélection naturelle. Une explication plausible pourrait être que la plupart des femelles sont des individus à croissance lente avec une forte fécondité, ce qui peut devenir encore plus important dans un contexte de déclin de la population.

Afin d'explorer la question sur la persistance d'individus à croissance lente ou rapide au cours des générations, un modèle multigénérationnel démo-génétique à l'échelle de l'aire de distribution est nécessaire. Cela permettrait d'étudier les conditions écologiques dans lesquelles la plasticité phénotypique et le polymorphisme génétique peuvent être sélectionnés comme réponse à l'hétérogénéité environnementale et d'aborder l'échelle de la population plutôt que l'échelle du bassin versant. Cela pourrait en outre permettre d'étudier les conditions du maintien de ces mécanismes dans un contexte actuel de populations effondrées. Les conditions écologiques à 
explorer sont: le gradient de température entre les bassins versants (comme un gradient latitudinal qui représente l'aire de distribution des anguilles), la variabilité spatiale dans les bassins versants (c'est-à-dire le gradient en amont et l'aval) et les paramètres démographiques, tels que l'abondance de la population. Pour analyser l'émergence évolutive de la plasticité phénotypique, il faudrait également explorer l'effet de ses coûts, car ils constituent des contraintes majeures pour l'avantage sélectif de ce mécanisme.

La capacité d'adaptation des anguilles est basée sur la combinaison de la diversité génétique et la plasticité phénotypique qui sont source de la diversité des traits d'histoire de vie et permettent aux anguilles d'utiliser différents habitats. De ce fait, les anguilles représentent des cas d'étude intéressants sur comment la diversité peut contribuer à la résilience et à la stabilité de la population. La grande variabilité des réponses aux conditions environnementales pendant la phase continentale suggère que les mesures de gestion et de conservation devraient chercher à préserver la diversité des habitats, la diversité génétique et la diversité des traits de vie (Secor, 2015b). Ces diversités sont primordiales puisqu'ils sont source de portfolio et de storage effects (ICES, 2009; Secor, 2015a).

Un nombre important d'espèces est menacé par le changement global. Dans ce contexte, il faut améliorer les connaissances sur la capacité des espèces à l'adaptation et leur vitesse d'adaptation pour orienter des stratégies de gestion et des politiques de conservation. Ces dernières années, de nombreuses études ont abordé ce sujet, en se focalisant sur les mécanismes évolutifs et les avantages de la sélection par rapport à la plasticité phénotypique sur le taux d'adaptation. $\mathrm{Ce}$ travail contribue à ce débat en utilisant les anguilles à titre d'exemple.

\section{REMERCIEMENTS}

Cette étude a été financée par l'équipe commune de recherche HYNES EDF-Irstea. Nous tenons à remercier les deux relecteurs de ce travail qui ont grandement permis de l'améliorer. Nous remercions Laurent Beaulaton, Martin Castonguay, Bruno Ernande et Christian Rigaud pour leur soutien pendant ce travail de thèse. Nous remercions également Emmanuelle Cam, Eric Feunteun, Marie Nevoux, Etienne Rivot et David Secor pour leurs réflexions au cours de la soutenance de thèse dont a fait l'objet ce travail.

\section{RÉFÉRENCES}

Acou A., Lefebvre F., Contournet P., Poizat G., Panfili J. \& Crivelli A.J., 2003. Silvering of female eels (Anguilla anguilla) in two sub-populations of the Rhône Delta. Bull. Fr. Pêche Piscic. 368: 55-68.

Andrello M., Bevacqua D., Maes G.E. \& De Leo G.A., 2011. An integrated geneticdemographic model to unravel the origin of genetic structure in European eel (Anguilla anguilla L.). Evol. Appl. 4: 517-533.

Belpaire C., Pujolar J.M., Geeraerts C. \& Maes G.E., 2016. Contaminants in eels and their role in the collapse of the eel 
stocks. In: Biology and Ecology of Anguillid eels (T. Arai, Ed.), pp. 225250. Florida: Boca Raton.

Bevacqua D., Andrello M., Melia P., Vincenzi S., De Leo G.A. \& Crivelli A.J., 2011a. Density-dependent and interspecific interactions affecting European eel settlement in freshwater habitats. Hydrobiologia 671: 259-265.

Bevacqua D., Melià P., de Leo G.A. \& Gatto M., 2011b. Intra-specific scaling of natural mortality in fish: The paradigmatic case of the European eel. Popul. Ecol. 165: 333-339.

Boivin B., Castonguay M., Audet C., Pavey S.A., Dionne M. \& Bernatchez L., 2015. How does salinity influence habitat selection and growth in juvenile American eels Anguilla rostrata? J. Fish Biol. 86: 765-784.

Brodersen J. \& Seehausen O., 2014. Why evolutionary biologists should get seriously involved in ecological monitoring and applied biodiversity assessment programs. Evol. Appl. 7: 968-983.

Byer J.D., Lebeuf M., Trottier S., Raach M., Alaee M., Stephen Brown R., Backus S., Casselman J.M. \& Hodson P.V., 2015. Trends of persistent organic pollutants in American eel (Anguilla rostrata) from eastern Lake Ontario, Canada, and their potential effects on recruitment. Sci. Total Environ. 529: 231-242.

Cairns D.K., Secor D.A., Morrison W.E. \& Hallett J.A., 2009. Salinity-linked growth in anguillid eels and the paradox of temperate-zone catadromy. J. Fish Biol. 74: 2094-2114.

Castonguay M., Hodson P.V., Couillard C. M., Eckersley M.J., Dutil J.D. \& Verreault G., 1994a. Why is recruitment of the American eel, Anguilla rostrata, declining in the St. Lawrence River and Gulf? Can. J. Fish. Aquat. Sci. 51: 479-488.
Castonguay M., Hodson P.V., Moriarty C., Drinkwater K.F. \& Jessop B.M., 1994b. Is there a role of ocean environment in American and European eel decline? Fish. Oceanogr. 3: 197-203.

Côté C.L., Castonguay M., Verreault G. \& Bernatchez L., 2009. Differential effects of origin and salinity rearing conditions on growth of glass eels of the American eel Anguilla rostrata: Implications for stocking programmes. J. Fish Biol. 74: 1934-1948.

Côté C.L., Castonguay M., McWilliam K.S., Gordon C. \& Bernatchez L., 2014. In absence of local adaptation, plasticity and spatially varying selection rule: $A$ view from genomic reaction norms in a panmictic species (Anguilla rostrata). BMC Genomics 15: 403.

Côté C.L., Pavey S.A., Stacey J.A., Pratt T. C., Castonguay M., Audet C. \& Bernatchez L., 2015. Growth, female size, and sex ratio variability in American eel of different origins in both controlled conditions and the wild: Implications for Stocking Programs. Trans. Am. Fish. Soc. 144: 246-257.

Daverat F., Limburg K., Thibault I., Shiao J.C., Dodson J., Caron F., Tzeng W.-N., lizuka Y. \& Wickström H., 2006. Phenotypic plasticity of habitat use by three temperate eel species, Anguilla anguilla, $A$. japonica and $A$. rostrata. Mar. Ecol. Prog. Ser. 308: 231-241.

Daverat F., Beaulaton L., Poole R., Lambert P., Wickstrom H., Andersson J., Aprahamian M., Hizem B., Elie P., YalcinOzdilek S. \& Gumus A., 2012. One century of eel growth: Changes and implications. Ecol. Freshw. Fish 21: 325-336.

Davey A. \& Jellyman D., 2005. Sex determination in freshwater eels and management options for manipulation of sex. Rev. Fish Biol. Fish. 15: 37-52. 
De Leo G.A. \& Gatto M., 1995. A size and age-structured model of the European eel (Anguilla anguilla L.). Can. J. Fish. Aquat. Sci. 52: 1351-1367.

Dekker W., 1998. Long-term trends in the glasseels immigrating at Den Oever, The Netherlands. Bull. Fr. Pêche Piscic. 199-214.

Dekker W., 2000. A Procrustean assessment of the European eel stock. ICES J. Mar. Sci. 57: 938-947.

Dekker W., 2003a. On the distribution of the European eel (Anguilla anguilla) and its fisheries. Can. J. Fish. Aquat. Sci. 60: 787-799.

Dekker W., 2003b. Status of the European eel stock and fisheries. In: Eel biology (K. Aida, K. Tsukamoto, K. Yamauchi, Eds.), pp. 237-254. Tokyo, Japan: Springer-Verlag.

Dekker W. \& Casselman J.M., 2014. The 2003 Québec Declaration of Concern about eel declines-11 years later: Are eels climbing back up the slippery slope? Fisheries 39: 613-614.

Dekker W., Casselman J.M., Cairns D.K., Tsukamoto K., Jellyman D. \& Lickers H., 2003. Québec Declaration of Concern: Worldwide decline of eel resources necessitates immediate action. Fisheries 28: 181.

Desaunay Y. \& Guerault D., 1997. Seasonal and long-term changes in biometrics of eel larvae: A possible relationship between recruitment variation and North Atlantic ecosystem productivity. J. Fish Biol. 51: 317-339.

Desaunay Y., Lecomte-Finiger R. \& Guérault $D ., 2012$. Mean age and migration patterns of Anguilla anguilla (L.) glass eels from three French estuaries (Somme, Vilaine and Adour Rivers). Arch. Pol. Fish. 20: 185-190.

Drouineau H., Rigaud C., Daverat F. \& Lambert P., 2014. EvEel (evolutionary ecologybased model for eel): A model to explore the role of phenotypic plasticity as an adaptive response of three temperate eels to spatially structured environments. Can. J. Fish. Aquat. Sci. 71: 1561-1571.

Edeline E., 2007. Adaptive phenotypic plasticity of eel diadromy. Mar. Ecol. Prog. Ser. 341: 229-232.

Ernande B. \& Dieckmann U., 2004. The evolution of phenotypic plasticity in spatially structured environments: Implications of intraspecific competition, plasticity costs and environmental characteristics. J. Evol. Biol. 17: 613-628.

Feunteun E., 2002. Management and restoration of European eel population (Anguilla anguilla): An impossible bargain. Ecol. Eng. 18: 575-591.

Gagnaire P.-A., Normandeau E., Côté C., Hansen M.M. \& Bernatchez L., 2012. The genetic consequences of spatially varying selection in the panmictic American eel (Anguilla rostrata). Genetics 190: 725-736.

Geffroy B. \& Bardonnet A., 2012. Differential effects of behaviour, propensity to migrate and recruitment season on glass eels and elvers' growing performance. Ecol. Freshw. Fish 21: 469482.

Geffroy B. \& Bardonnet A., 2016. Sex differentiation and sex determination in eels: Consequences for management. Fish Fish. 17: 375-398.

Helfman G., Bozeman E. \& Brothers E., 1984. Size, age, and sex of American eels in a Georgia river. Trans. Am. Fish. Soc. 113: 132-141.

Helfman G., Facey D.E., Stanton Hales L. Jr., \& Bozeman E.L. Jr., 1987. Reproductive ecology of the American eel. Am. Fish. Soc. Symp. 1: 42-56.

Ibbotson A., Smith J., Scarlett P. \& Aprhamian M., 2002. Colonisation of freshwater habitats by the European eel Anguilla anguilla. Freshw. Biol. 47: 1696-1706. 
ICES., 2009. Report of the Study Group on Anguillid Eels in Saline Waters (SGAE$S A W)$. Sackville, Canada-Gothenburg, Sweden.

Iman R.L. \& Conover W.J., 1980. Small sample sensitivity analysis techniques for computer models. with an application to risk assessment. Commun. Stat.Theory Methods 9: 1749-1842.

Jacoby D. \& Gollock M., 2014a. Anguilla anguilla. The IUCN red list of threatened species. Version 2014. 2. Available from http://www.iucnredlist.org.

Jacoby D. \& Gollock M., 2014b. Anguilla japonica. The IUCN red list of threatened species. Version 2014. 2. Available from http://www.iucnredlist.org.

Jacoby D., Casselman J.M., DeLucia M., Hammerson G.A. \& Gollock M., 2014. Anguilla rostrata. The IUCN red list of threatened species. Version 2014. Available from http://www.iucnredlist. org.

Jacoby D.M.P., Casselman J.M., Crook V., DeLucia M.-B., Ahn H., Kaifu K., Kurwie T., Sasal P., Silfvergrip A.M.C., Smith K. G., Uchida K., Walker A.M. \& Gollock M. J., 2015. Synergistic patterns of threat and the challenges facing global anguillid eel conservation. Glob. Ecol. Conserv. 4: 321-333.

Jessop B.M., 2010. Geographic effects on American eel (Anguilla rostrata) life history characteristics and strategies. Can. J. Fish. Aquat. Sci. 67: 326-346.

Kettle A.J., Asbjørn Vøllestad L. \& Wibig J., 2011. Where once the eel and the elephant were together: Decline of the European eel because of changing hydrology in southwest Europe and northwest Africa? Fish Fish. 12: 380411.

Koops M.A., Young A. \& Cairns D.K., 2014. Meta-population dynamics in the american eel and the importance of larval distribution. Afs.
Levins R., 1963. Theory of fitness in a heterogeneous environment. II. Developmental flexibility and niche selection. Amercian Nat. 47: 75-90.

Lobón-Cerviá J. \& Iglesias T., 2008. Longterm numerical changes and regulation in a river stock of European eel Anguilla anguilla. Freshw. Biol. 53: 1832-1844.

Mateo M., Lambert P., Tétard S., Castonguay M., Ernande B. \& Drouineau H., 2017a. Cause or consequence? Exploring the role of phenotypic plasticity and genetic polymorphism in the emergence of phenotypic spatial patterns of the European eel. Can. J. Fish. Aquat. Sci. 74: 987-999.

Mateo M., Lambert P., Tétard S. \& Drouineau $H_{\text {., }} 2017 \mathrm{~b}$. Impacts that cause the highest direct mortality of individuals do not necessarily have the greatest influence on temperate eel escapement. Fish. Res. 193: 51-59.

McCleave J., 1993. Physical and behavioural controls on the oceanic distribution and migration of leptocephali. J. Fish Biol. 43: 243-273.

McKay M.D., 1988. Sensitivity and uncertainty analysis using a statistical sample of input values. Uncertain. Anal. 145186.

McKay M.D., Beckman R.J. \& Conover W.J. 2000. A comparison of three methods for selecting values of input variables in the analysis of output from a computer code. Technometrics 42: 55-61.

Melià P., Bevacqua D., Crivelli A., Panfili J., De Leo G. \& Gatto M., 2006a. Sex differentiation of the European eel in brackish and freshwater environments: A comparative analysis. J. Fish Biol. 69: 1228-1235.

Melià P., Bevacqua D., Crivelli A.J., De Leo G.A., Panfili J. \& Gatto M., 2006b. Age and growth of Anguilla anguilla in the Camargue lagoons. J. Fish Biol. 68: 876-890. 
Oliveira K., 1999. Life history characteristics and strategies of the American eel, Anguilla rostrata. Can. J. Fish. Aquat. Sci. 56: 795-802.

Oliveira K. \& McCleave J., 2000. Variation in population and life history traits of the American eel, Anguilla rostrata, in four rivers in Maine. Environ. Biol. Fishes 59: 141-151.

Oliveira K., McCleave J. \& Wippelhauser G., 2001. Regional variation and the effect of lake: River area on sex distribution of American eels. J. Fish Biol. 58: 943-952.

Pavey S.A., Gaudin J., Normandeau E., Dionne M., Castonguay M., Audet C. \& Bernatchez L., 2015. RAD Sequencing Highlights Polygenic Discrimination of habitat ecotypes in the panmictic American eel. Curr. Biol. 26: 1666-1671.

Pujolar J.M., Bevacqua D., Capoccioni F., Ciccotti E., De Leo G.A. \& Zane L., 2011. No apparent genetic bottleneck in the demographically declining European eel using molecular genetics and forward-time simulations. Conserv. Genet. 12: 813-825.

Pujolar J.M., Jacobsen M.W., Als T.D., Frydenberg J., Munch K., Jónsson B., Jian J.B., Cheng L., Maes G.E., Bernatchez L. \& Hansen T., 2014. Genomewide single-generation signatures of local selection in the panmictic European eel. Mol. Ecol. 23: 2514-2528.

Saltelli A., Tarantola S. \& Campolongo F., 2000. Sensitivity analysis as an ingredient of modeling. Stat. Sci. 377-395.

Schmidt J., 1923. Breeding places and migrations of the eel. Nature 111: 51-54.

Secor D., 2015a. Migration Ecology of Marine Fishes. Baltimore: Johns Hopkins University Press, 304 p.
Secor D.H., 2015b. American Eel: When does diversity matter? Fisheries 40: 462-463.

Smogor R.A., Angermeier P.L. \& Gaylord C. K., 1995. Distribution and abundance of American eels in Virginia streams: Tests of null models across spatial scales. Trans. Am. Fish. Soc. 124: 789-803.

Tesch F.W., 2003. The Eel. Oxford, UK: Blackwell Publishing, $408 \mathrm{p}$.

Tsukamoto K., 1992. Discovery of the spawning area for Japanese eel. Nature 356: 789-791.

Vélez-Espino L.A. \& Koops M.A., 2009. A synthesis of the ecological processes influencing variation in life history and movement patterns of American eel: towards a global assessment. Rev. Fish Biol. Fish. 20: 163-186.

Vladykov V.D., 1966. Remarks on the American Eel (Anguilla rostrata LeSueur). Sizes of elvers entering streams; the relative abundance of adult males and females; and present economic importance of eels in North America. Verhandlungen Int. Ver. Für Theor. Angew. Limnol. 16: 1007-1017.

Vøllestad L.A., 1992. Geographic variation in age and length at metamorphosis of maturing European eel-Environmental effects and phenotypic plasticity. J. Anim. Ecol. 61: 41-48.

Vøllestad L.A. \& Jonsson B., 1988. A 13year study of population dynamics and growth of the European eel Anguilla anguilla in a Norwegian River: Evidence for density-dependent mortality, and development of a model for predicting yield. J. Anim. Ecol. 57: 983-997. 